American Journal of Applied Sciences 7 (6): 732-738, 2010

ISSN 1546-9239

(C) 2010Science Publications

\title{
Focusing of the Flow Capture for Local Exhaust Ventilation Systems
}

\author{
${ }^{1}$ S.Y. Spotar and ${ }^{2}$ A.L. Sorokin \\ ${ }^{1}$ Department of Chemical and Environmental Engineering, Faculty of Engineering, \\ The University of Nottingham, Malaysia Campus, Semenyih, Malaysia \\ ${ }^{2}$ Institute of Thermophysics Siberian Branch of Russian Academy of Science, Novosibirsk, Russia
}

\begin{abstract}
Problem statement: The extraction hoods commonly used as inlet element of the local ventilation systems exhibit limited emission capture over moderate distances from the source of the hazardous emissions. Proposed inclusion of a swirling peripheral jet was found to increase the effective length over which the extraction hood successfully captures harmful fumes. However information on a detailed pattern field of the vortex focused inflow was insufficient thus restricting the potential applications of the method. Approach: The numerical modeling study of the focusing by vortex inflow was accomplished to reveal the implication of the key operating parameters. In addition the visualization technique was applied to confirm the fume capturing features. Results: The simulated overall flow field patterns for an inflow of $10 \mathrm{~m} \mathrm{sec}^{-1}$ value under sets of 0.5-1.5 swirl numbers and 0$21 \mathrm{~m} \mathrm{sec}^{-1}$ outcome velocities of peripheral jet showed the arrangement and contour of the capture stream. Under optimum parameters the capture zone derived from the vector velocity field yielded up to 4 inlet diameters near-axis distance from the extractor entrance. The complimentary observations using laser sheet visualization technique confirmed the enhanced capturing capacity from the mist or smoke sources of emission. Conclusion: Through numerical modeling study the capacity of peripheral vortex shielding to generate the more concentrated exhaust inflow comparing with action of the conventional hood has been elucidated.
\end{abstract}

Key words: Capturing flow, vortex suction, local ventilation, numerical modeling

\section{INTRODUCTION}

Industrial local exhaust ventilation systems employ the concept of removing contaminants at or near its source or point of generation to prevent its release into the workspace environment (ACGIH, 2004; Davis, 2000). The standard conventional hoods do not perform satisfactory, especially in cases where emission sources are distanced from the suction aperture due to space requirements of the technological operation. Generally, from standard fluid mechanics (Batchelor, 2000) the inflow induced with conventional hood represents an inherently unfocused inviscid sink-type flow in contrast with rather concentrated viscid jet-type flow.

Previous study (Spotar et al., 1995) proposed the application of a peripheral swirl jet to act as a separating boundary zone between the extracted fumes and the surrounding atmosphere upstream of the extractor entrance. Termed the Vortex Suction Device (VSD) it was found to focus the flow of contaminants away from the source and into the extractor. The vortex suction method uses the high ejection features of the swirling radial jet that implicitly shields the exhaust duct. The resulting overall flow consists of (a) peripheral swirling radial jet, (b) upward exhaust flow directed to the mouth or duct entrance and (c) low velocity demarcation buffer zone in between. The appropriate vortex suction device comprises of circular and annular ducts, a tangential duct, a vane type vortex generator and an ultradiffusor employed to produce the unlocked vortex jet operation mode due to Coanda effect. As the additional peripheral airstream only assists to concentrate an exhaust flow thus the improving efficiency of the entire local ventilation system it doesn't request increasing of the appropriate filter unit capacity.

Whereas the pattern of the inflow induced with a hood is properly investigated (Cascetta, 1996) the velocity fields of the flow generated VSD are described only conceptually which might restrict practitioners to estimate the potential of VSD application. Hence the main objective of this study is to investigate the details of the flows generated with VSD under set of the key governing parameters.

Corresponding Author: S.Y. Spotar, Department of Chemical and Environmental Engineering, Faculty of Engineering, The University of Nottingham, Malaysia Campus, Semenyih, Malaysia 
The numerical modeling of the VSD induced flows has been carried out using FLUENT 6.3 software to illustrate the flow field present for various operating parameters and ultradiffusor design. Work is also qualitatively compared to experiments using flow visualization techniques.

\section{Flow modeling:}

Flow organization scheme: A schematic illustration of the flow producing arrangement of the VSD is shown in the Fig. 1. An inflow of $10 \mathrm{~m} \mathrm{sec}^{-1}$ velocity value was set at the entrance to the exhaust duct of inner diameter (d) equal to $100 \mathrm{~mm}$ and outer Diameter (D) equal to $130 \mathrm{~mm}$. The ultradiffusor had a curvature of $r_{c}=26 \mathrm{~mm}$. It is assumed that the inflow velocity contour (capturing region) is determined by the velocity limit of $0.2 \mathrm{~m} \mathrm{sec}^{-1}$. (Here the inflow velocity is attributed to the negative scale). An external boundary of the modeled flow space was designated at a sufficient distance from the exhaust aperture, in this case by setting the operating space boundary as a $1 \mathrm{~m}$ radius sphere centered on the VSD. It was assumed that the sphere surface can be treated as 'infinity' that reflects the real insensibility of the VSD induced capturing contour to the unspecified particulars of external environment flow configurations with arbitrary small scale air velocity distribution. The modeling aimed to observe the flow configuration development under variation of the peripheral air flow rate, intensity of swirl and shape of the ultradiffusor while the exhaust air flow rate was kept constant. The swirl intensity of outcoming jet $\Omega=\overline{\mathrm{w}}_{\mathrm{j}} / \overline{\mathrm{u}}_{\mathrm{j}}$ was defined here as a ratio of the tangential velocity over axial velocity. Commonly swirl is created by passing the peripheral flow through the swirl vanes arrangement.

FLUENT options setup: Non-uniform meshing of the space was generated with provision for the wall boundary layer and trailing edge flow effects. The generated mesh consisted of approximately 20000 quadrilateral cells with minimum size of $0.4 \mathrm{~mm}$ in vicinity of trailing edge and maximum size of $15-20 \mathrm{~mm}$ at the far-field flow zones. The configuration of the mesh in the region adjacent to the VSD is shown in Fig. 2. The following key settings of FLUENT options have been applied: Solver-pressure based, formulationimplicit, space-axisymmetric swirl, time-steady, gradient option-Green-Gauss cell based, turbulence model-Reynolds stress model.

The boundary conditions were defined for the swirl peripheral jet at leaving end of the guide vanes assembly as 'velocity inlet' option, for the surrounding flux at the external boundary of the sphere space as 'velocity inlet option' and for the inflow inside the exhaust duct as 'outflow' option. The outcoming peripheral flow was set by the values of axial mean velocity value $\overline{\mathrm{u}}_{\mathrm{j}}$ and swirl intensity $\Omega$.

The value of a mm $\sec ^{-1}$ scale air velocities at the external boundary were estimated from the entire space volumetric flow rate balance subject to the value of mean exhaust velocity of $\bar{u}_{\mathrm{s}}=10 \mathrm{~m} \mathrm{sec}^{-1}$.

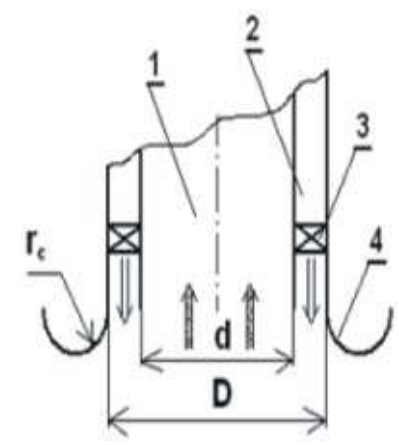

Fig. 1: Basic components of the vortex suction device: (1) Exhaust duct; (2) Coaxial channel for incoming air; (3) Swirl vane generator; (4) Ultradiffusor

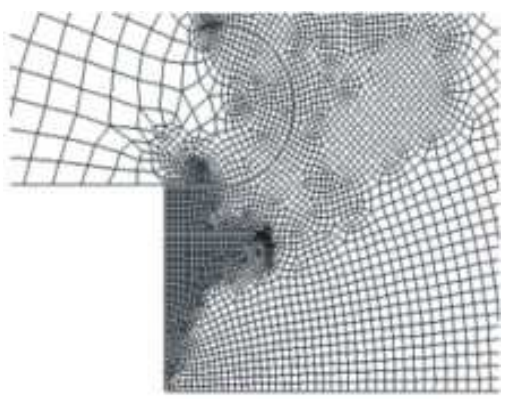

Fig. 2: Meshing structure near aperture of VSD

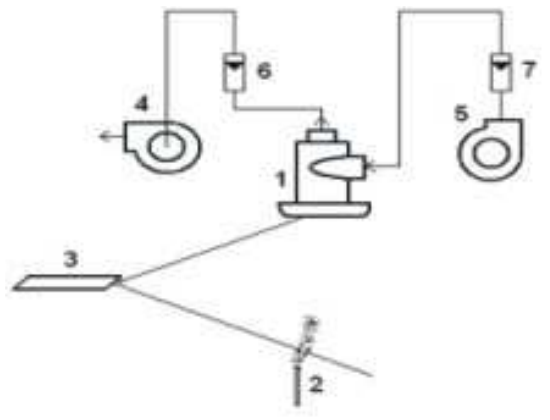

Fig. 3: Experimental set-up: (1) VSD unit; (2) Pointlike source of fume; (3) Laser sheet system; (4, 5) Air moving units; $(6,7)$ Rotameters 


\section{MATERIALS AND METHODS}

Experiment: The schematic of the experimental facility used in this study is depicted in Fig. 3. The airflow streams through both the exhaust and blowing circuits were created by consumer vacuum cleaners powered from voltage-controlled current sources. The air flowrates were set via voltage variation and measured with rotameters.

The VSD unit used in the experiments is characterized by the following parameters: $\mathrm{d}=30 \mathrm{~mm}$, $\mathrm{D}=40 \mathrm{~mm}, \mathrm{r}_{\mathrm{c}}=9 \mathrm{~mm}, \Omega \approx 1$. Either water mist generated using an ultrasonic atomizer or fumes from smoldering sticks were used as the light scattering "seeding" particles. A NEO-50MG green laser sheet system served as a light source for illumination.

\section{RESULTS}

From preliminary visual observations it was found that the optimum exhaust performance induced by VSD was attained under moderate swirl number $\Omega \approx 1$ with peripheral jet air flow rate being approximately the same as the exhaust inflow rate. To obtain a clearer

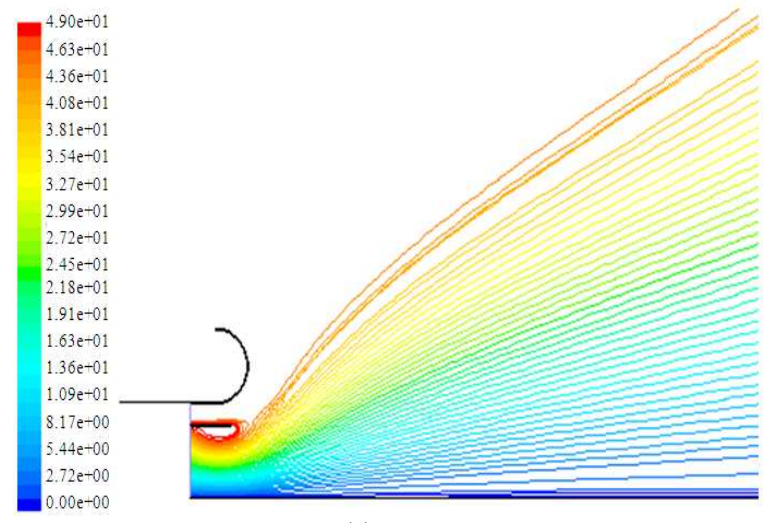

(a)

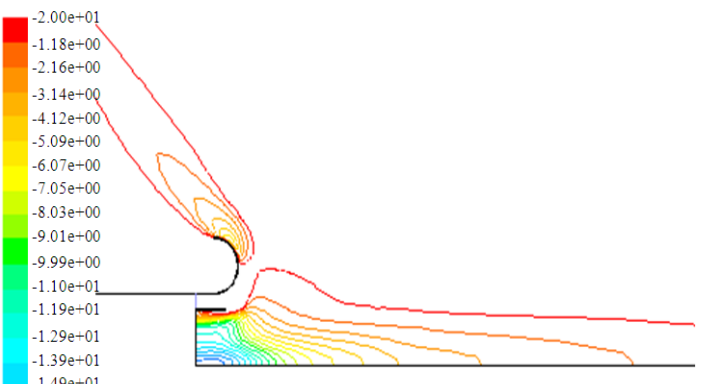

(c)

understanding into the flow fields present in the inflow, both with and without swirl, basic numerical modeling results are presented in Fig. 4-9.

The increase in focusing of inflow with the introduction of swirl is clearly revealed by comparing Fig. 4 and 5a, where streamlines of exhaust inflow are plotted.

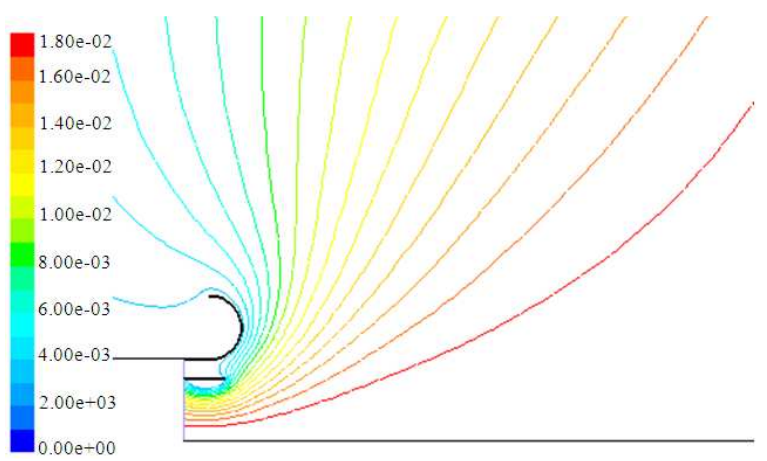

Fig. 4: Unfocused inflow streamlines corresponding to a sink type flow arrangement. $\overline{\mathrm{u}}_{\mathrm{s}}=10 \mathrm{~m} \mathrm{sec}^{-1}$, $\overline{\mathrm{u}}_{\mathrm{j}}=0 \mathrm{~m} \mathrm{sec}^{-1}$

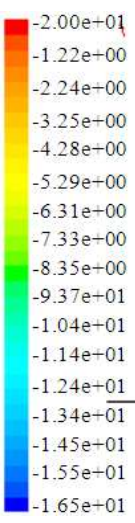

$1.65 e+01$

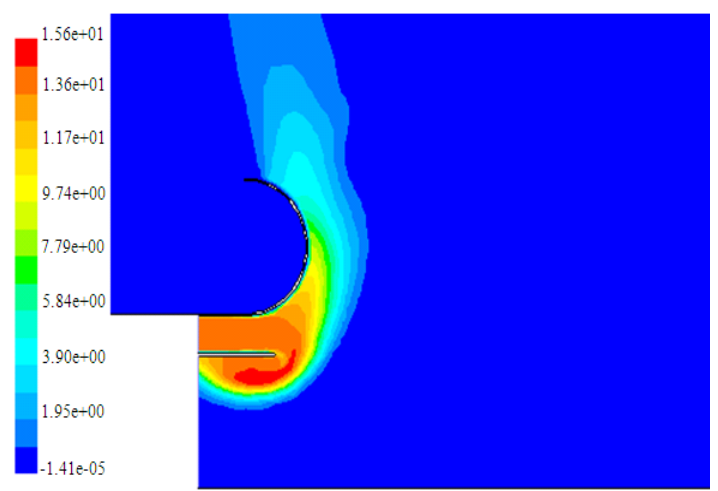

(d) 


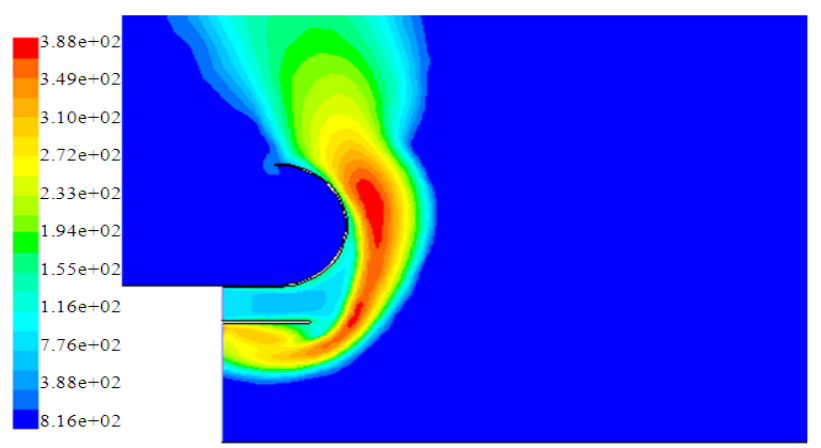

(e)

Fig. 5: (a) Inflow streamlines; (b), velocity vectors field; (c) contours of axial velocity; (d) contour of tangential velocity and (e) turbulent intensity under shielding effect of peripheral swirling jet. $\bar{u}_{\mathrm{s}}=10 \mathrm{~m} \mathrm{sec}^{-1}$, $\overline{\mathrm{u}}_{\mathrm{j}}=14.5 \mathrm{~m} \mathrm{sec}^{-1}, \Omega=1$. Axial velocity contours and velocity vector field are cut off by $-0.2 \mathrm{~m} \mathrm{sec}^{-1}$ axial velocity value

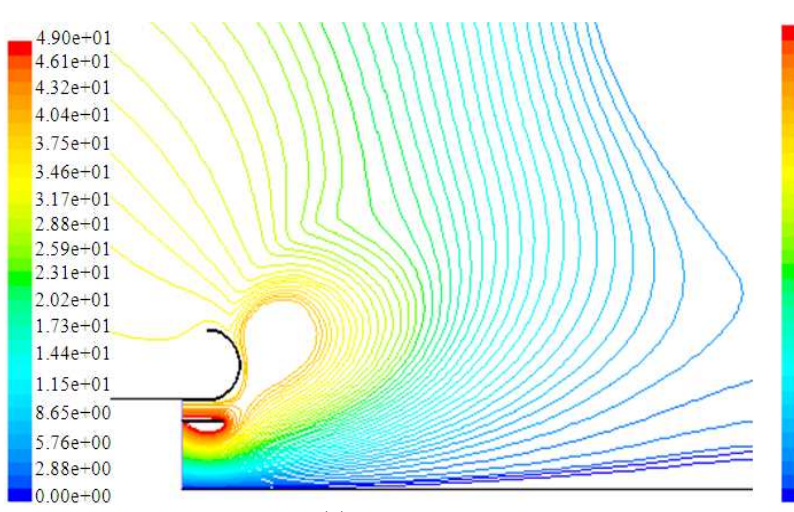

(a)

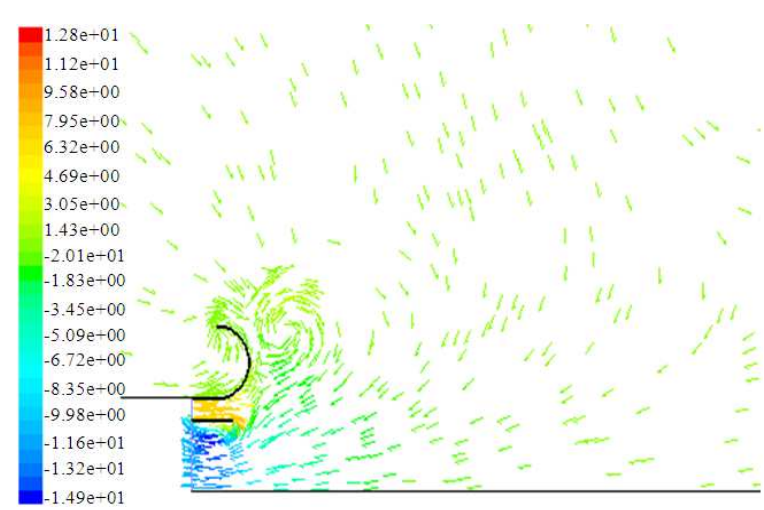

(b)

Fig. 6: (a) Exhaust inflow streamlines and (b) velocity vectors for overall flow (b). $\overline{\mathrm{u}}_{\mathrm{s}}=10 \mathrm{~m} \mathrm{sec}{ }^{-1}, \overline{\mathrm{u}}_{\mathrm{j}}=7 \mathrm{~m}$ $\sec ^{-1}, \Omega=1$

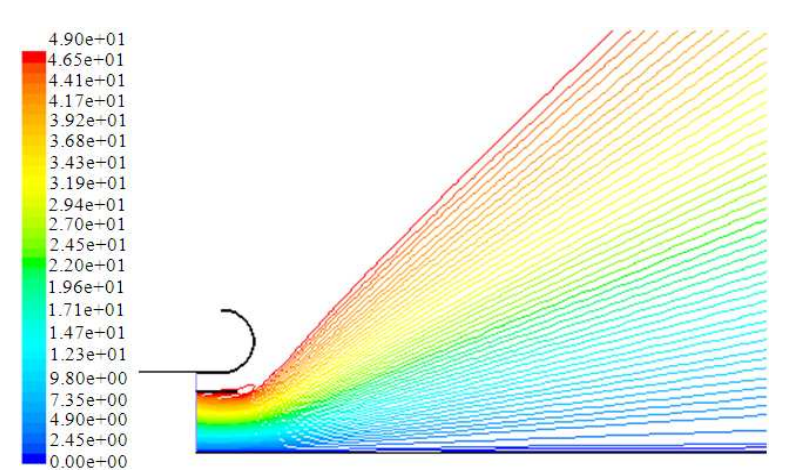

(a)

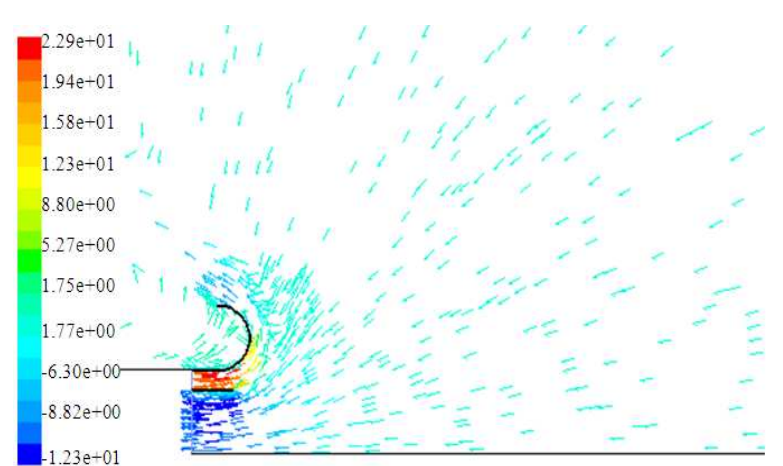

(b)

Fig. 7: (a) Exhaust inflow streamlines and (b) velocity vectors for overall flow (b). $\bar{u}_{\mathrm{s}}=10 \mathrm{~m} \mathrm{sec} \mathrm{se}^{-1}, \overline{\mathrm{u}}_{\mathrm{j}}=21 \mathrm{~m}$ $\sec ^{-1}, \Omega=1$ 


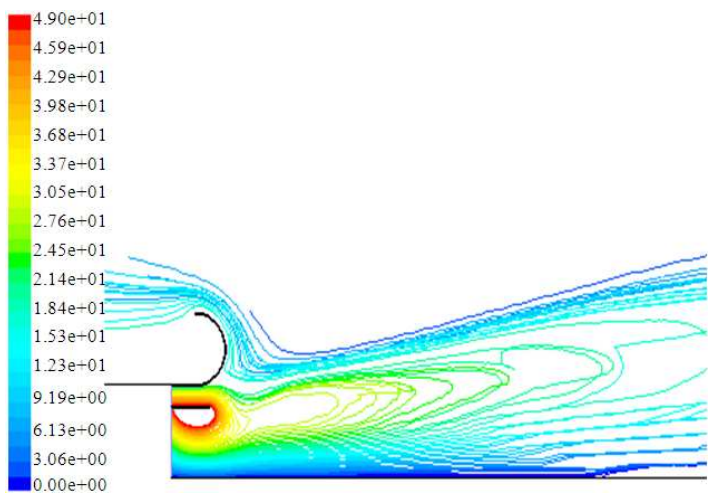

(a)

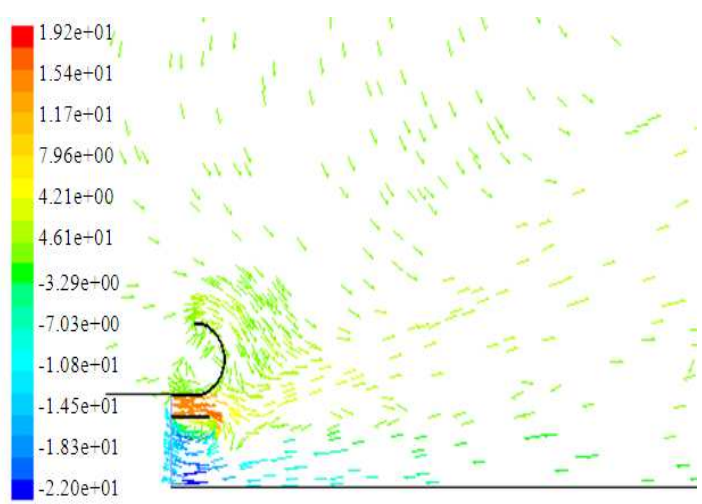

(b)

Fig. 8: (a) Exhaust Inflow streamlines; (b) and velocity vectors for overall flow. $\overline{\mathrm{u}}_{\mathrm{s}}=10 \mathrm{~m} \mathrm{sec}{ }^{-1}, \overline{\mathrm{u}}_{\mathrm{j}}=14.5$ $\mathrm{m} \mathrm{sec}^{-1}, \Omega=0.5$

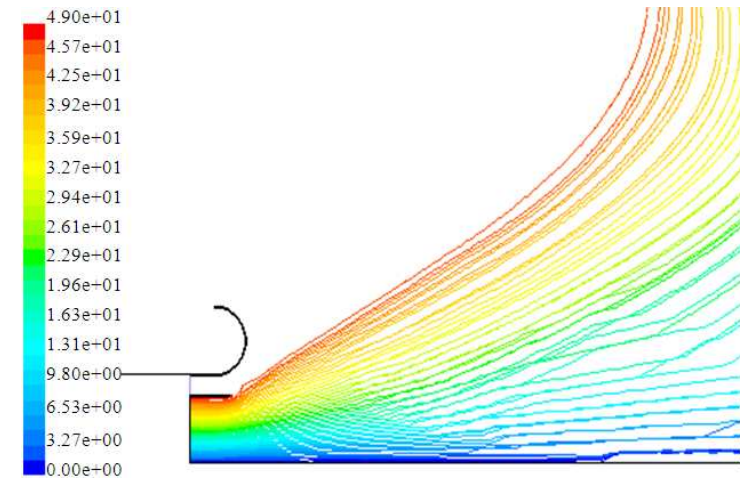

(a)

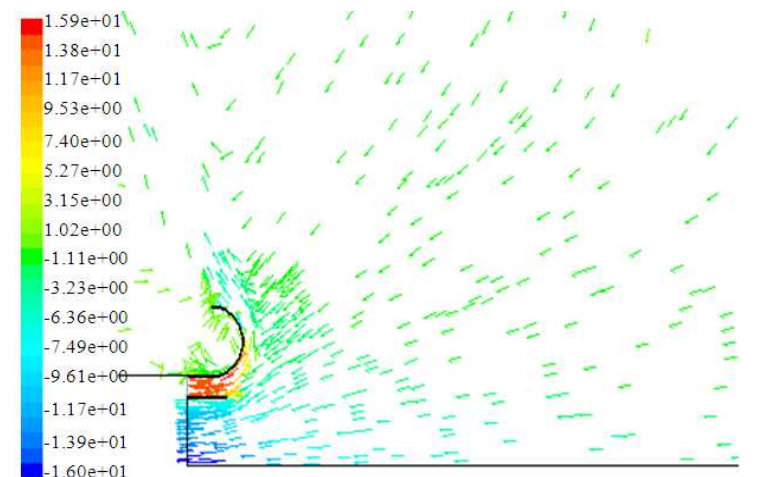

(b)

Fig. 9: (a) Exhaust inflow streamlines and (b) velocity vectors for overall flow. $\overline{\mathrm{u}}_{\mathrm{s}}=10 \mathrm{~m} \mathrm{sec}{ }^{-1}, \overline{\mathrm{u}}_{\mathrm{j}}=14.5 \mathrm{~m}$ $\sec ^{-1}, \Omega=1.5$

Here the inflow means namely only the flow being sucked into the entrance of the ventilation system. The flow presented by Fig. 4 corresponds to the typical sink type unfocused inflow, while the VSD creates near-axis concentrated inflow due to the shielding action of the swirling peripheral jet. The extension of the capturing zone is traced from Fig. 5b-c where the plotted regions of velocity vector field and contours of axial velocity are purposely cut off by the specified axial velocity value which is $-0.2 \mathrm{~m} \mathrm{sec}^{-1}$ and thereby yielding approximately $4 \mathrm{~d}$ near-axis capturing distance from the extractor entrance.

An increase in peripheral flow above that used for the optimal arrangement corresponded to Fig. 5a does not yield further exhaust inflow flow focusing (Fig. 7). This illustrates that above a certain peripheral flow rate there is little increase in flow focusing. The complementary velocity vector field (Fig. 7b) shows that the observation in Fig. 7a is due to the high peripheral jet flow rate enhancing the off-axis bypass flow past the inlet of the extraction duct.

The effect of the swirl intensity is recognized through the flow patterns given by Fig. 5, 8 and 9. The imparted angular momentum flux in combination with translating Coanda effect ultradiffusor diverts the peripheral jet in the radial direction preventing immediate interaction with the exhaust inflow. In particular Fig. 8 clearly demonstrates that under insufficient swirl the peripheral outcoming jet is captured into the exhaust inflow.

Impact of the high swirl number (Fig. 9, $\Omega=1.5$ ) on the flow configuration is analogous to the case of the excessive flow rate of peripheral jet of moderate swirl $\Omega=1$ (Fig. 7), however it is associated with a high pressure drop for air flow passing through the swirl vanes assembly. 


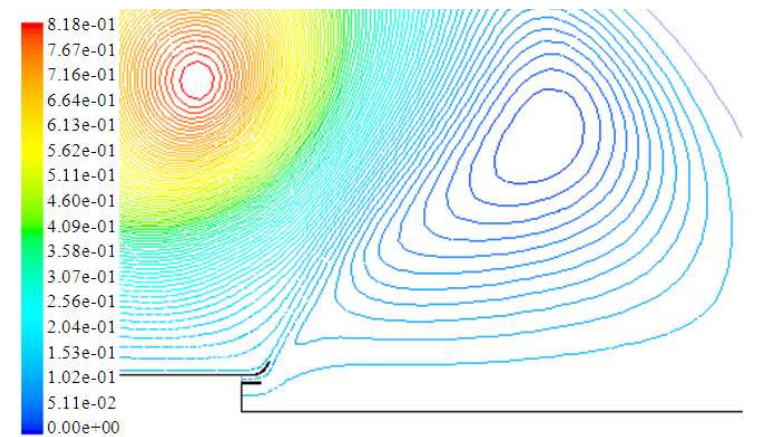

Fig. 10: Overall flow streamlines in the vicinity of for the VSD equipped with a quarter circle profiled diffuser. $\overline{\mathrm{u}}_{\mathrm{s}}=10 \mathrm{~m} \mathrm{sec}{ }^{-1}, \overline{\mathrm{u}}_{\mathrm{j}}=14.5 \mathrm{~m} \mathrm{sec}^{-1}$, $\Omega=1$

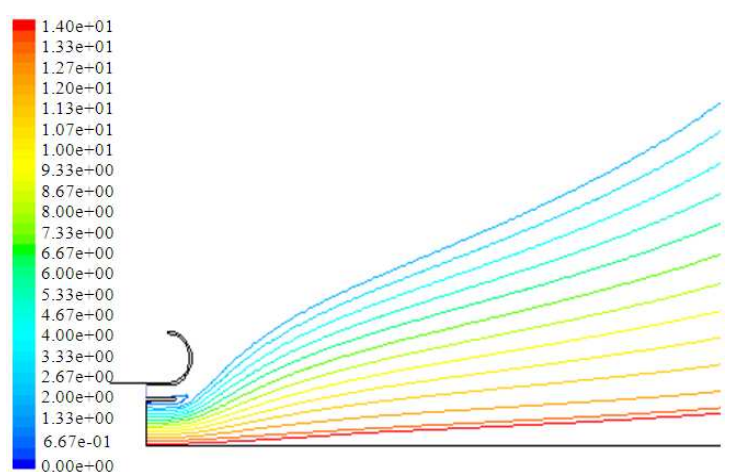

(a)

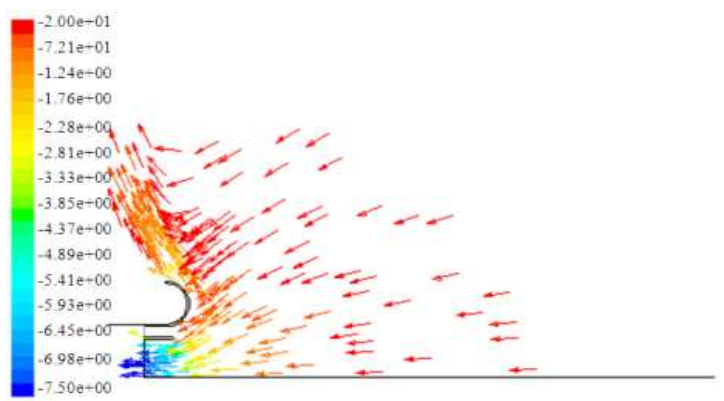

(b)

Fig. 11: (a) Inflow streamlines and (b) velocity vector fields for the VSD units use in experiments ( $d$ $=30 \mathrm{~mm}, \mathrm{D}=40 \mathrm{~mm}) . \quad \overline{\mathrm{u}}_{\mathrm{s}}=6 \mathrm{~m} \mathrm{sec} \mathrm{se}^{-1}, \overline{\mathrm{u}}_{\mathrm{j}}=$ $9 \mathrm{~m} \mathrm{sec}^{-1}, \Omega \approx 1$. Velocity vectors field are cut off by $-0.2 \mathrm{~m} \mathrm{sec}^{-1}$ axial velocity value

Figure 10 displays overall flow streamlines for the VSD equipped with a modified shape of the diffuser attempting to send an outgoing recirculation jet at a $60^{\circ}$ angle to the inflow. Flow simulation predicts that a substantial part of the inflow is formed into a torodial circulation cell structure.

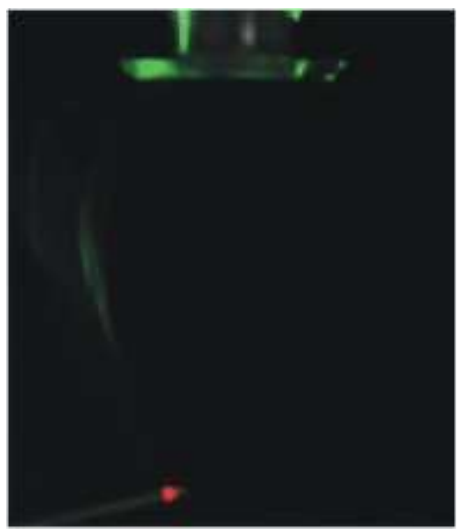

(a)

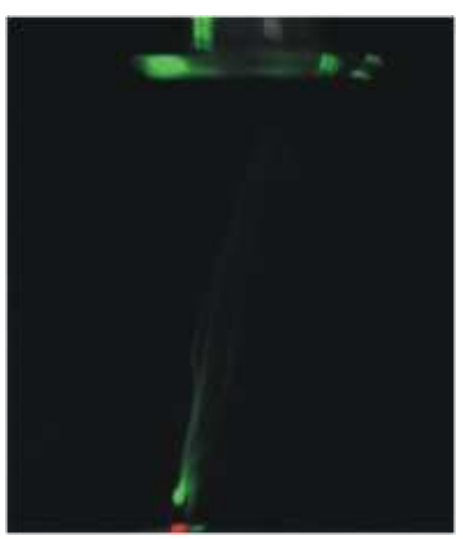

(b)

Fig. 12: (a) Fume goes in uncertain direction under sink type inflow $\overline{\mathrm{u}}_{\mathrm{s}}=6 \mathrm{~m} \mathrm{sec}^{-1}, \overline{\mathrm{u}}_{\mathrm{j}}=0 \mathrm{~m} \mathrm{sec}^{-1}$; (b) fume is conveyed to the VSD aperture due to action of the peripheral jet. $\overline{\mathrm{u}}_{\mathrm{s}}=6 \mathrm{~m} \mathrm{sec}^{-1}$, $\overline{\mathrm{u}}_{\mathrm{j}}=9 \mathrm{~m} \mathrm{sec}^{-1}(\mathrm{~d}=30 \mathrm{~mm}, \mathrm{D}=40 \mathrm{~mm}), \Omega \approx 1$

The numerical modeling was also performed for the smaller VSD unit used in the experiment sets yielding the flow structure (Fig. 11), similar to those given in the Fig. 5.

Examples of the experimental findings are given in Fig. 12 where the fume from the smoldering stick positioned at $\sim 4 \mathrm{~d}$ distance from the aperture. With the introduction of peripheral flow the inflow is more focused towards the extraction system entry and no aerosols bypass the device and remain in the open atmosphere.

\section{DISCUSSION}

A negligible part of the peripheral jet is sucked at once into the exhaust aperture yielding some minor 
angular momentum into the external part of exhaust flow (Fig. 5d). It might be possible to reduce this undesirable effect by variation of design parameters but it is unlikely to be possible to suppress this effect completely. The high turbulent intensity is developed close to the ultradiffusor (Fig. 5e) while near-axis flow is characterized by low turbulence intensity which should prevent the dispersion of captured contaminants into surrounding.

The reduction in flow rate in the peripheral jet (Fig. 6) leads to the formation of a flow pattern similar to that seen for no swirl i.e., a sink-type inflow. In this case the peripheral jet is too weak to produce segregation between the inflow and the surrounding fluid.

There is fast decay of swirl for all cases, thus the regions of noticeable tangential velocity component are localized mainly within the peripheral jet outlet like demonstrated in the Fig. 5d.

It was observed that under the normal operation mode the peripheral jet is deflected by the half circle profiled diffusor in radial direction (at $90^{\circ}$ angle) according the prediction however a complete $\mathrm{U}$-turn for the peripheral jet similar to given in the Fig. $7 \mathrm{~b}$ was not registered in the experiments, giving earlier flow separation still at $\sim 90^{\circ}$ angle.

The modeling for the VSD equipped with a quarter circle profiled diffusor gave acceptable flow structure with an appropriate inflow focusing effect; however in experiments it was found that such a mode is unstable and somehow recaptured into a suction flow. Such instability is often interpreted as a manifestation of multiple possible solutions for the vortex flows however such discussion is beyond the scope of this study. Reliable predictions of separation effects presumably require more advanced analysis for assumptions and numerical procedures.

Thus steady occurrence of the toroidal recirculation cell (Fig. 10) in reality is questionable and future study will look at visualizing these conditions experimentally.

\section{CONCLUSION}

Through numerical modeling procedures the capacity of VSD to generate the more concentrated exhaust inflow comparing with action of the conventional hood has been elucidated. Such concentrated inflow makes possible the fume capturing from the extended distance in application to the pointlike or localized sources of emission. Presented data on the structure of the VSD induced flow yield the key information that in combination with characteristics of the source of contaminants provides a means for evaluation of tackling a given emission control problem by the vortex suction method.

\section{REFERENCES}

ACGIH, 2004. Industrial Ventilation: A Manual of Recommended Practice. 25th Edn., ACGIH, ISBN: 978-1-882417-52-0 2004, pp: 350.

Batchelor, G.K., 2000. An Introduction to Fluid Mechanics. Cambridge University Press, ISBN: 13: 978-0521663960, pp: 635.

Cascetta, F., 1996. Experimental evaluation of the velocity fields for local exhaust hoods with circular and rectangular openings. Build. Environ., 31: 437-449. DOI: 10.1016/S0360-1323(00)00087-1

Davis, W.R., 2000. Air Pollution Engineering Manual by Air and Waste Management Association. 2nd Edn., Wiley, John and Sons, Inc., ISBN: 13: 9780471333333 pp: 912.

Spotar, S.Y., I.A. Chokhar, V.V. Lukashov and D.S. Prozorov, 1995. Method and device for local ventilation. Russ. Patent. No. 2046258. 